\title{
Transposition
}

Musique et Sciences Sociales

$8 \mid 2019$

Musique : patrimoine immatériel ?

\section{Claude Coste et Sylvie Douche (éds.), Barthes et la musique}

Rennes, PUR, 2018

\section{Cécile Raulet}

\section{(2) OpenEdition}

\section{Journals}

Édition électronique

URL : http://journals.openedition.org/transposition/3018

DOI : 10.4000/transposition.3018

ISSN : 2110-6134

\section{Éditeur}

CRAL - Centre de recherche sur les arts et le langage

Référence électronique

Cécile Raulet, "Claude Coste et Sylvie Douche (éds.), Barthes et la musique », Transposition [En ligne], 8| 2019, mis en ligne le 15 septembre 2019, consulté le 17 décembre 2020. URL : http://

journals.openedition.org/transposition/3018; DOI : https://doi.org/10.4000/transposition.3018

Ce document a été généré automatiquement le 17 décembre 2020.

La revue Transposition est mise à disposition selon les termes de la Licence Creative Commons Attribution - Partage dans les Mêmes Conditions 4.0 International. 


\section{Claude Coste et Sylvie Douche (éds.), Barthes et la musique}

Rennes, PUR, 2018

Cécile Raulet

\section{RÉFÉRENCE}

Claude Coste et Sylvie Douche (éds.), Barthes et la musique, Rennes, PUR, 2018, 362 p.

Ce que je vais tenter de dire du « grain » ne sera, bien sûr, que le versant apparemment abstrait, le compte rendu impossible d'une jouissance individuelle que j'éprouve continûment en écoutant chanter ${ }^{1}$.

1 La musique est chez Roland Barthes, de manière plus ou moins entremêlée selon les textes, ce qu'il aime, ce qu'il connaît, ce dont il connaît l'histoire, ce dont il défend une connaissance et une pratique autres que celles qui l'entourent ou prévalent, ce dont il cherche à parler, ce avec quoi il parle, ce avec quoi il pense, ce qu'il utilise pour transmettre une expérience, ce qui (lui) permet de parler de littérature et de ce qu'il veut penser, avec ce qu'il aime (parce qu'il l'aime, parce que cela lui donne à penser). L'ensemble formé par les contributions du collectif Barthes et la musique, travaillant en somme les différentes valeurs du et conjoignant un auteur et ce qui est pour lui plus qu'un objet d'étude et de critique, permet de lier ces différents aspects - et démontre, si cela était nécessaire, la richesse et les cohérences d'une œuvre qui, lue à travers un prisme, ouvre à des pistes variées, fait résonner la musique avec d'autres problématiques propres à son auteur (certains contributeurs se livrent d'ailleurs au bel exercice de faire parler des textes ne traitant pas frontalement de musique, et montrent aussi que les textes sur la musique peuvent parler (aider à parler) d'autre chose). 
2 Dirigé par Claude Coste et Sylvie Douche, Barthes et la musique est né d'un colloque éponyme dans le cadre du riche centenaire, en 2015, de la naissance de Roland Barthes; l'agencement des contributions a été passé au filtre d'une réorganisation faisant de l'ensemble une intéressante croisée des chemins, à partir d'un objet auquel les contemporains de Barthes se sont peu attachés, d'un thème assez peu traité par les barthésiens. Le volume réunit barthésiens et musicologues, qui ont pour la plupart accepté de dialoguer avec Barthes, et sur d'autres aspects de son œuvre, plutôt que d'en discuter les propositions musicologiques stricto sensu, ce qui aide à la richesse du livre.

Du point de vue de barthésienne depuis lequel il a été lu, Barthes et la musique a donc une véritable valeur en tant que volume, dans la mesure où il rend compte de moult facettes de l'objet "Barthes et la musique», et donne les clefs, nombreuses et essentielles, sur le sujet. En premier lieu, le recueil embrasse le corpus concerné, à la fois restreint en volume - quelques articles et passages thématisant la musique ${ }^{2}$ - et étendu : dans le temps, puisque la musique traverse toute l'œuvre, et dans la variété des interventions autant que des propos, puisque la musique irradie dans toute l'œuvre de Barthes. En second lieu, les contributions - d'apport par ailleurs inégal mais possédant presque toutes une force d'interrogation, d'entraînement à interroger plus avant les textes commentés - montrent bien que la musique est un élément important de la vie quotidienne de Barthes : il la pratique (piano et chant, selon les périodes de sa vie, à quoi s'ajoute une connaissance de la théorie et de l'histoire de la musique) et défend fermement, quoique dans les faits cette hiérarchie soit atténuée, la primauté de la musique jouée sur la musique écoutée ${ }^{3}$. On perçoit bien également, à lire Barthes et la musique, que, dans sa vie créatrice, la musique constitue pour Barthes un référent et une référence pour le discours, ainsi que ce que Françoise Escal a appelé un «formant, [un] schème compositionnel ${ }^{4}$ ", et un conducteur pour la pensée.

Barthes et la musique, c'est d'abord Barthes s'attelant à construire un objet intellectuel pour travailler à son intelligibilité, autant que questionner et défendre la nature affective et donc singulière des rapports de tel sujet à un tel objet. Comme recueil, Barthes et la musique rend compte, mais épars, de nombre d'apports barthésiens concernant la musique stricto sensu; je donnerai donc une trop réductrice liste des propositions ayant la musique pour objet de réflexion immédiat, que viendront compléter celles évoquées au fil du détail des contributions.

La musique est chez Barthes le lieu par excellence de participation, d'expression et de liberté du corps; les goûts musicaux, très sélectifs, à la fois historiquement et sociologiquement marqués (il y a chez Barthes une importante conscience de l'historicité de l'interprétation), sont intellectuellement défendus comme goûts ${ }^{5}$ : "Ordre manifeste, impérieux, du aimer/ne pas aimer: la musique. [Ordre de la valeur absolue, ou plutôt de la valeur absolument manifestée] », écrit Barthes dans ses fiches personnelles ${ }^{6}$. En somme, parler de musique, ce sera traiter du pour-moi de telle œuvre (de telle œuvre pour moi), plutôt que de la signification (à valeur absolue) de celle-ci. Barthes, à partir de ce qui constitue bien un critère d'évaluation, revisite expressivité et pathos; ce, notamment par une réflexion sur le grain de la voix, pensé dans cet « espace (genre) très précis où une langue rencontre une voix ${ }^{7}$ » qu'est pour Barthes la musique chantée : le grain de la voix, c'est «la matérialité du corps parlant sa langue maternelle ", qui ne se réduit pas au timbre, qui relève du géno-chant opposé au phénochant $^{8}$; le " "grain", c'est le corps dans la voix qui chante, [...] dans le membre qui exécute ${ }^{9}$ ". 
Barthes réfléchit encore à l'intérêt de la stéréophonie, à la force critique de l'intermezzo, produit des réflexions sur le chant romantique, notamment sur la manière dont celui-ci active et fait jouer chez le récepteur le fantasme d'un " corps unifié $^{10} »$; ou encore, il pose des jalons pour articuler sensible et intelligible, déjouer le paradigme entre " pure sensualité » et ordre tout puissant du développement ${ }^{11}$.

7 La musique pour Barthes est encore l'un des écrins permettant de vivre, penser et décrire la signifiance. C'est aussi pourquoi, comme expérience, elle aide à penser la littérature, et en particulier le Texte, à y rêver voire entendre une "musique du sens $^{12}$ ». L'histoire de la musique joue là un rôle (la mélodie française a pour Barthes "travaillé la langue à travers le poème ${ }^{13}$ "); si telle œuvre, tel courant, sont tirés du côté de la théorie du texte, ils n'en sont pas dénaturés pour autant : c'est encore (aussi) de musique qu'il est question lorsque Barthes pratique ce genre de déports.

8 Parce que la valeur dépend du goût, il est fort possible que, bien qu'absolue pour un sujet, elle ne soit que peu partagée; "valeur injustifiable, car il n'y a pas de critique musicale $^{14} »$, conclut la note barthésienne citée plus haut. Et pourtant, partant du constat d'une prégnance de la «critique adjective (ou [de] l'interprétation prédicative) ", Barthes interroge bien la critique musicale en tant qu'elle est une activité d'écriture, et s'interroge sur la possibilité de la pratiquer autrement: "plutôt que d'essayer de changer directement le langage sur la musique, il vaudrait mieux changer l'objet musical lui-même, tel qu'il s'offre à la parole : modifier son niveau de perception ou d'intellection : déplacer la frange de contact de la musique et du langage ${ }^{15}$ ».

Dans le même temps, Barthes travaille à une certaine transmission de ses goûts - qui acquièrent alors une force d'enseignement double, puisque le discours malgré tout produit véhicule telle conception, singulière, de tel compositeur ou œuvre, et une réflexion (réflexive) sur l'évaluation. L'expérience musicale (que Barthes pense à partir de celle de Schumann) ne relève pas du domaine de l'intelligible mais « de ce qui bat dans le corps » et partant d'un corps « qui bat ${ }^{16}$ » - encore cette matière peut-elle être dite, rendue intelligible, c'est une grande composante de l'art barthésien.

10 Tout cela pour rappeler, ou faire connaître, partiellement, la variété des chemins que pouvaient emprunter les contributeurs s'attelant au sujet «Barthes et la musique ». Cerner l'activité qui consiste à écouter, penser ce qui du corps d'un compositeur bat dans sa musique, interroger sa propre pratique musicale, se rendre à même de comprendre ce qui point ou non à l'écoute d'une œuvre ou d'une interprétation, le tout sciemment articulé à une subjectivité dont la conception est en travail constant : on voit que les idées avancées par Barthes sur la musique relèvent du domaine de la pratique. C'est sur cette dernière que Claude Coste et Sylvie Douche, qui ont dirigé Barthes et la musique, ont décidé d'axer leur introduction (digne de ce nom, puisque ses auteurs ne se contentent pas de présenter les contributions, mais bien d'introduire, à leur manière, savante et sensible à Barthes, au sujet qu'ils proposent).

11 Ce même fil structure le volume: la première partie porte sur la pratique de composition et de jeu de Barthes ; la seconde sur celle du commentaire : critique écrite, notations plus ou moins développées lors d'entretiens et commentaire très personnel de la musique aimée à la radio ; la troisième se penche sur la musique chez Barthes en tant qu'elle est un « objet d'inspiration, un modèle pour toutes les formes de création » (p.11). " Si la musique se décline au propre et au figuré, si elle irrigue toute l'œuvre, c'est qu'elle est profondément liée au processus de vie »(p.12) : la quatrième partie fait jouer de multiples manières « la musique, la vie », et accorde une place particulière à la 
voix (qui, dans les jeux de présence et d'absence qu'elle active, permet d'articuler "matérialité du présent» et " conscience tragique de l'existence »). La cinquième et dernière partie fait entrer Barthes en dialogue avec d'autres figures produisant une pensée de la musique, à partir d'une pratique de la musique elle-même, de l'écriture, ou de la philosophie.

\section{Pratique}

Le volume comme ensemble permet donc encore de prendre la mesure de l'intrication fertile des différentes pratiques barthésiennes concernant la musique. Pour détailler certains des apports de Barthes et la musique, on suivra ce fil des pratiques, mais selon un ordre autre, afin de tâcher de montrer autrement encore la fertilité du volume (et, parce que celle-ci est réelle et fait la qualité de l'ensemble, on se permet de regretter un nombre certain d'erreurs dans la transcription des citations et des entretiens, dans les renvois aux œuvres, ainsi que quelques menus problèmes de langue).

Le volume s'ouvre sur la pratique de la musique au sens le plus strict du terme, à savoir le jeu et la composition. Sylvie Douche propose non un simple compte-rendu de la production de Barthes (une quinzaine de pièces entre 1934 et 1941, composées au piano, et «majoritairement pensées pour piano»), mais une véritable analyse faisant voir chez Barthes compositeur des constantes, et une personnalité. Cette dernière est plutôt de joueur amateur que de compositeur, ce qui ancre avec profit la figure de l'amateur, très en vue chez les barthésiens, dans la pratique de la composition même. Douche à la fois dépouille et analyse les archives, décrit la musique composée, établit des liens entre créations et données physiques (les mouvements de la main) et plus largement biographiques, et relie la production musicale de Barthes à ses autres pratiques artistiques.

Analysant le "discours musical de Barthes", le travail de Sylvie Douche présente encore l'intérêt d'un rapprochement avec l'écriture (les premiers textes coïncident avec la fin de l'activité de composition) et en particulier avec les "stylèmes" barthésiens, tel travail court donc aussi les risques inhérents à l'acte d'accoler à la création musicale la grille de lecture d'une autre pratique; sont ainsi mis en regard développement sur ostinato et spirale, développement thématique limité, pièces courtes et fragment. Plus qu'entre écriture musicale et écriture "verbale », le parallèle véritablement fécond est sans doute entre musique composée - expérimentée - et commentaire (notamment de la musique) : cette approche gagne toute sa force lorsque des liens sont esquissés entre des dissonances, voulues par Barthes mais jugées incongrues par Douche, et ce qui sera dit plus tard par l'écrivain lui-même de la musique et en particulier de la tonalité et de la dissonance.

\section{Pratiques culturelles}

Certaines contributions s'attèlent aux pratiques culturelles de Barthes: Hervé Lacombe détaille les modalités et explore les possibles raisons d'une absence de goût de Barthes pour l'opéra, peut-être son rejet, en tout cas un intérêt " différé, médié, compliqué » (p. 101). Ses pratiques culturelles rendent compte de ses goûts, pensés par Barthes comme irréductibles et ne devant pas être éteints, puisqu'ils sont ce avec quoi il écrit (avec quoi il faut écrire). Ce qu'illustre Ralph Heyndels, qui, à partir du texte de 
Barthes de 1978 sur le Palace, mythique music-hall, théâtre et haut lieu de la nuit parisienne, écrit sur une "présence absente ", sur l'absence de la musique dans un texte sur un lieu où elle est pourtant vive et présente. Heyndels aborde donc indirectement la double question $d u$ goût et des goûts de Barthes, à travers celle de la situation dans laquelle se trouve l'écrivain au moment de composer son texte.

La centralité de ses goûts personnels dans la production de Barthes se perçoit très bien quand Florence Fabre analyse l'«affinité profonde et intime" qui unit Barthes à Schumann. Schumann, pour Barthes et chez Barthes, c'est à la fois ce qu'il aime, ce avec quoi il entretient un contact physique et ce qui lui fait percevoir au mieux la vie du corps dans l'œuvre, ce qui lui permet de comprendre ce qu'il aime dans la musique, celui qui pratique des formes aimées (on notera un entrecroisement fécond : ces formes sont aimées et défendues parce que pratiquées par Barthes lui-même, appréciées parce qu'elles lui permettent l'exercice d'une projection légère en même temps que d'une réflexivité détournée, et désirées sous la forme du modèle en même temps que de l'utopie). Fabre prend également à bras-le-corps la problématique question de l'analogie entre une écriture musicale aimée et une écriture verbale (voir les p. 63-68).

17 À travers la figure de l'amateur, Barthes propose une conception générale et singulière de la pratique (à laquelle il ne faut cependant pas donner l'exclusivité). Coste et Douche rappellent que l'amateurisme, dont Barthes fait « une philosophie et une poétique ", « n'exclut nullement l'art savant et l'inventivité la plus inouïe » (p. 17-18), on voudrait ajouter : ni non plus la rigueur de la pensée autant que de la création. L'amateur n'est pas nécessairement dilettante, ni en demi-teinte, et tout en lui est actif. Pour Mathias Écœur, cette figure, «avant d'être déplacée, reportée vers les objets les plus divers, se forge dans l'expérience musicale - c'est-à-dire précisément dans une pratique - et, plus tard, se précise dans les quelques écrits portant sur la musique » (p. 170); si l'on veut envisager toute la portée d'une telle figure, notons qu'il faut cependant, complétant immédiatement ce rôle séminal de la musique, prendre en considération l'intrication des pratiques artistiques qui fait aussi l'originalité et la force de la figure barthésienne de l'amateur ${ }^{17}$.

"Celui qui à la fois aime et fait » (p. 170) est donc nécessairement praticien, engage son corps. La figure est également politique, car il y a une "gratuité de la pratique de l'amateur, au sens où sa production est soustraite à tout impératif d'échange » (p. 174) Écœur montre plus largement que la musique, comme pratique et comme objet de réflexion, participe de la lutte contre les travers bourgeois, notamment la logique de consommation et la passivité que celle-ci implique (ce qui explique encore que la musique joue un rôle crucial dans le renouvellement de la conception du lecteur, et une réflexion pédagogique).

Plus imagées, et devant néanmoins toujours autant à la musique, sont les attentions portées aux manières de jouer, produire et écrire de Barthes. Si François Noudelmann débute son essai en soulignant la séparation - défendue par Barthes afin, selon Noudelmann, «de préserver le domaine du jeu de la prose savante »- de la musique jouée et de la musique dont on parle, la suite entremêle à raison le jeu musical et le jeu de la production intellectuelle. Ce point est illustré par l'objet même de la contribution, le doigté, qui renvoie à une certaine discipline de jeu (et une critique de l'asservissement des corps qui a elle aussi pour nom discipline), en même temps qu'à " une phénoménologie, une psychologie, une érotique, une politique et une éthique " (p. 54). Noudelmann articule sens premier et force métonymique du doigt, du tact, de la 
pression, de la mesure, et pratique, héritage proprement barthésien, l'amphibologie qui fait le sens fructueux.

Diana Knight, elle, s'attache à la musicalité, à la force musicale, de l'écriture de Barthes (en fait à sa manière une pratique musicale), à partir de "l'air barthésien » que constituent les notes du Journal de deuil, «composées entre la mort de sa mère et la sienne ». À partir d'un texte de Barthes écrit pour soi (pour soi seul, non destiné à publication ${ }^{18}$ ) et de l'œuvre de Barthes une fois endeuillé, l'auteure propose elle-même une belle variation autour de la lettre $r$ et du mot air, repère jeux d'homophonie et de formes, ainsi que quelque chose comme des refrains (et donc une rythmicité).

\section{Parole}

Si écouter de la musique est une activité solitaire en ce qu'elle met en jeu l'individu singulier, avec son affectivité propre, faire écouter ce que l'on aime, c'est prouver autrement qu'écouter constitue un acte autant qu'une attitude, qu'écouter, c'est faire. Guido Mattia Gallerani pose avec justesse que « la radio est le lieu d'un renversement de l'écoute de la musique. C'est le passage à une pratique différente de celle de l'amateur de musique, mais une pratique de la musique tout de même » (p. 154), dont le cadre est celui d'une " écoute collective » (p. 160); dans ses interventions radiophoniques autour de la musique, Barthes démontre donc que l'extrême singularité des goûts musicaux n'exclut pas un relatif partage.

Jean-Claire Vançon fournit une analyse des deux émissions de France Culture dans lesquelles est intervenu Barthes («l'objet [...] est constitué par l'invité qu'elles accueillent plus que par la musique qu'elles font entendre »); le dispositif - la personnalité « établit une programmation "égoïstement" conforme à ses goûts les plus chers » (p.134) - entre en adéquation avec les positions autant que l'ethos de Barthes, telle est sans doute la clé de la fertilité de telles expériences. La nature de ce que l'on tire à « écouter Roland Barthes écouter " est double : «ces émissions permettent à qui les écoute d'en apprendre sur Roland Barthes - notamment la manière dont le concept de "moire", central dans sa pensée des années 1970, prend probablement racine dans une expérience musicale de la tonalité " (c'est l'objet principal de l'article de Vançon) et "sur la musique que Barthes fait entendre, grâce à la langue dont le critique fait usage» (p. 143 ; je souligne). Écouter Barthes écouter, c'est donc aussi le regarder faire, l'entendre dire autrement que comme il écrit, retrouver encore la question de la possibilité de dire (la musique, le reste): Vançon livre une analyse (riche aussi de ce qu'elle interroge la parole barthésienne au moyen d'outils barthésiens) de la manière dont Barthes parle de la musique, et, ici surtout, de morceaux précis ; ce, grâce à l'étude d'une langue qui «met l'auditeur en situation d'entendre ce à quoi il n'aurait pas sans cela été nécessairement sensible, et sait mobiliser, non seulement l'attention de cet auditeur, mais aussi tout son corps.» Je ferai cette remarque que l'expérience radiophonique alliant Barthes et la musique ouvre à la question d'une transmission de la musique, et peut pour cela être éclairée par la conception du rôle, de la fonction et de la vertu critiques de Barthes: on dira que, comme le critique littéraire propose une lecture, la "parole de l'homme qui écoute ${ }^{19}$ " oriente l'auditeur: Barthes infléchit un temps l'écoute par une "langue qui donne la responsabilité du sens à celui qui l'écoute» (p. 142). En 1963, Barthes demandait à la critique qu'«elle affiche ses choi $^{20} »$, toujours idéologiques, ce qui est faisable littérairement, en en travaillant la 
formulation: «le critique affronte un objet qui n'est pas l'œuvre, mais son propre langage. Quel rapport un critique peut-il avoir avec le langage ? C'est de ce côté qu'il faut chercher la "subjectivité" du critique ${ }^{21} »$; en 1978-1979, à la radio, c'est d'une certaine façon tout ce pourquoi on le convie, et ce qu'il pratique, rendant un principe critique effectif, tout en lui imprimant une torsion, musicale.

\section{Écoute}

On l'a mentionné, Barthes formule une conception de l'écoute qui, si on la fait rejaillir sur les modes de lecture, de la partition comme du livre, invite plus largement à renouveler le rapport aux œuvres, le regard que l'on porte sur elles, la manière d'en parler; de l'écoute, on peut faire une façon de porter attention, d'exercer une attention de nature singulière dirigée vers les œuvres.

Yves Citton démontre à sa manière la cohérence de l'œuvre de Barthes en suivant tout au long de celle-ci une même intuition, à savoir "une certaine conception de l'expérience esthétique et de la vie des œuvres d'art, articulée par une dynamique attentionnelle dont l'article "Musica Practica" (1970) donnera tardivement la description la plus essentielle » (p. 185). L'attention est un faire, du côté de la praxis, qui suppose également de faire attention à ; elle fait jouer des liens variés entre présent et passé, implique le corps; le "faire attentionnel» implique le sujet, «me permet d'habiter activement » le temps, notamment par des détours salvateurs, qui déroutent l'attention et la rendent autrement productive, suivant des rythmes inédits. Citton propose donc de penser avec Barthes une "dynamique attentionnelle», qui est aussi une certaine éthique, au sens large: une manière de se tenir dans le monde, une "participation active et créative, dont la performance instrumentale constitue le modèle » (p. 185). Partant d'une lecture fidèle de Barthes, qui a travaillé à reconfigurer le «fossé entre créateurs et récepteurs, operatores et spectatores » (p. 199), Citton, porté par ses interrogations personnelles, s'écarte de Barthes, mais montre aussi ce que l'on peut en faire: que l'on adhère ou non à ses questionnements et approche, à un vocabulaire et un système de représentation très personnels, le propos porte incontestablement un regard inédit sur l'œuvre barthésienne et formule une proposition importante.

Pour Barthes, l'écoute n'est pas la simple «venue d'un signifié », il en fait quelque chose d'actif, et qui «oblige le sujet à renoncer à son "intimité" »; "l'écoute parle», mais aussi, on lui demande de "laisser surgir ${ }^{22}$ ».

François Balanche propose une lecture croisée de «Barthes selon Boucourechliev selon Barthes ", fidèle à une lecture mutuelle, à un croisement des pensées; Balanche pratique donc une histoire intellectuelle qui est moins celle des influences (décernant des primautés chronologiques) que celle d'une fécondité des penseurs d'une même période, d'une fécondation des idées des uns par celles des autres, la mise en lumière d'une «Weltanschauung partagée » (p. 320). Barthes et Boucourechliev se rejoignent sur la difficulté de parler de la musique et de nombreux autres points, et Balanche élit parmi eux celui de l'écoute. Il détaille les éléments d'une pensée de l'écoute, à la fois privée et dans l'espace social, selon trois axes - «reconsidération de l'écoute", « réhabilitation de l'auditeur » et « reconfiguration d'un monde » «à la mesure » de ces redéfinitions - qui dessinent ensemble un « fait musical total» (p. 310). 
Il en ressort que l'écoute chez Barthes comme chez Boucourechliev constitue un type d'attention qui est un faire - varié, car il dépend de ce qui est écouté -, un faire qui implique pleinement l'auditeur ${ }^{23}$, mais aussi « suppose et implique la réciprocité », du moins pose, et repose sur, un «espace intersubjectif». On relèvera la force politique d'une telle proposition, attachée au trouble voire au « renversement» des rapports de pouvoir qu'elle induit; on notera aussi le «terreau critique » dans lequel s'enracine la « reconfiguration de la société » qui va de pair avec les modifications de conception de l'écoute et de l'auditeur, et concerne bien sûr les pratiques culturelles. Les positions sur ces dernières, ainsi que sur l'exercice du goût d'une part, de l'analyse d'autre part, sont des points où les réflexions de l'écrivain et du compositeur se ressemblent moins qu'elles ne se complètent. Balanche ouvre également des pistes à partir de ce qui sépare Boucourechliev de Barthes et inversement, dans leur rapport respectif à la musique, différence due bien sûr à la position de l'un et l'autre vis-à-vis de celle-ci (en matière de musique, l'un est artiste, l'autre amateur).

\section{Pensée}

Barthes et la musique traite encore d'une autre pratique en nous renseignant sur la manière dont Barthes pense la musique. Ainsi, à travers une traversée de la production historique et critique sur les castrats, Timothée Picard montre que « la figure du castrat et la problématique de la voix sont largement prises dans des préoccupations de genres, de sexes et de sexualités » (p. 214), et met en lumière une spécificité de Barthes, chez qui le castrat ne tient pas, en tout cas pas en premier lieu, un rôle allégorique. "À la fois modèle et contre-modèle, désirable et terrifiant » (p. 222), le castrat de $S / Z$ apparaît comme un bon moyen d'articuler le double aspect des réflexions sur une telle figure, à savoir d'une part le pan psychanalytique de l'analyse des rapports des hommes homosexuels à l'opéra, d'autre part, plus visibles dans le livre de Barthes, les jeux de désexuation et de troubles dans le genre. On comprend que sur ces deux points et des questions adjacentes, $S / Z$ déplace les lignes, Barthes se place autrement.

Thomas Baldwin étudie de son côté la métaphore chez Proust telle qu'elle est pensée par Barthes, et la musique comme métaphore chez Barthes pour parler de Proust. Baldwin montre que l'idée de "variations sans thème " permet à Barthes de parler de Proust et du travail du critique : " pour Barthes, le critique n'est ni un interprète, ni un exécutant, ni un performeur des variations déployées dans le texte de Proust, mais un opérateur, l'on pourrait encore dire un auteur, des variations qu'il effectue sur celuici.» (p. 204). Baldwin développe ces idées, fécondes mais non développées par Barthes, à partir de textes de Proust et du séminaire (inédit) que Barthes lui a consacré à Rabat dans l'année 1969-1970; Baldwin ouvre à une réflexion (plutôt qu'il ne la développe, et ce qu'il en fait mériterait une véritable discussion) sur la manière de créer de Barthes lui-même, qui ne relève sans doute pas exactement des "variations sans thème ", mais me semble proche de ce que dit ailleurs Barthes de Proust, parlant d'une œuvre qui serait « une sorte de galaxie qui est infiniment explorable parce que les particules en changent de place et permutent entre elles » (cité p. 203).

Complétant un ensemble formé par les contributions faisant au passage sa place à Proust dans l'analyse et le commentaire de Barthes, s'appuyant, aussi, sur Proust, pour comprendre et développer des réflexions de Barthes, Éric Marty enrichit le dossier des rapports de Barthes à Proust, des différents rôles de Proust chez Barthes, en montrant 
comment ce dernier se démarque de l'un de ses grands textes tuteurs. Pour Marty, Barthes oppose à une certaine naïveté de Proust cet « objet musical supérieur » qu'est la gamme, et partant, au souvenir proustien, une "mémoire objective ou objectale " (p. 277). L'auteur pointe et démontre l'effort de Barthes - rare parmi les écrivains et penseurs de son époque - pour constituer la musique en objet (l'élever à la dignité d'objet). S'opère quelque chose comme une démythification qui permettrait précisément de parler de musique, avec justesse et rigueur.

31 Dans un article passionnant, Christophe Corbier travaille l'idée d'une autre histoire de la musique, organisée selon de nouveaux axes, aiguillée par d'autres objets ${ }^{24}$; celle-ci est traitée en filigrane tout au long d'un texte qui rend compte de glissements de Barthes l'amenant à penser la voix - « la voix, résume Corbier, c'est le signifiant qui se montre sans que l'expression lyrique prenne le dessus » (p. 86) -, à fonder cette histoire de la musique sur le corps. Au fil d'une histoire de la musique-chez/pour-Barthes, Corbier met donc en lumière l'articulation de la musique et des autres arts, notamment à partir de la décennie 1960 (et en particulier dans les Essais critiques (1964), ce qui mérite d'être relevé, puisque ceux-ci sont rarement abordés de ce point de vue par la critique barthésienne).

La pensée de Barthes sur la musique apparaît avec profit dans des rapprochements avec penseurs, écrivains, qui nous disent aussi comment pense Barthes et comment penser avec lui la musique.

Faisant bien sentir les nuances au sein d'un rapprochement très fondé, Yvan Nommick procède à une belle étude comparée de la " présence du piano dans la pensée musicale de Roland Barthes et de Vladimir Jankélévitch »; cette pensée a tout à voir avec la vie musicale de l'un et l'autre. La mise en regard des deux auteurs repose sur une pratique du piano telle qu'émerge une réflexion sur l'amateurisme ainsi que la mise en avant de l'écoute de soi-même jouant plutôt que celle des professionnels, parce que «la jouissance de la musique passe par la sensibilité tactile»(p.287), que prime «la musique avec laquelle on a un contact charnel » (p. 286). En commun également, le fait que le corpus de compositeurs commentés s'élabore selon goûts et affects plutôt que raison (p. 295). Enfin, l'importance de la pratique et ces points précis décident d'une pensée musicale qui est une pensée - commentaire et éventuelle théorisation - à partir de ce que l'un et l'autre aiment ou rejettent. Quant aux goûts eux-mêmes, une certaine communauté apparaît encore, puisque Barthes et Jankélévitch (bien que chacun à sa manière) «mettent en relief la façon dont leurs compositeurs aimés dépassent, ou quelquefois rejettent, les techniques de développement thématique motivique", expriment un "refus du développement discursif dans la composition musicale", apprécient et défendent la «juxtaposition rhapsodique» (p. 296); Barthes et Jankélévitch s'avèrent sur ce point des penseurs sensibles à et dans leur siècle, puisque la réflexion sur la musique au $\mathrm{xx}^{\mathrm{e}}$ siècle met au centre la question de la forme musicale (ce qui entre en résonance avec les pratiques de composition de Barthes lui-même décrites par Sylvie Douche).

Julien Labia propose une forme d'état des lieux (peut-être plus des pratiques que des idées) de la philosophie de la musique, cet état des lieux est fonction de Barthes, d'où sa vitalité. En retour, Labia procède à un début de mise en perspective (à vertu contextualisante comme projective) des réflexions de Barthes au regard de la philosophie de la musique (ce rapprochement fécond concerne principalement l'ontologie de la musique, mais aussi la pensée formaliste). Mais, s'il montre bien ce que 
Barthes peut apporter aux attitudes philosophiques (rapport à l'objet, forme d'implication, écriture), en quoi Barthes peut aider à penser philosophiquement, Labia traite finalement peu des propositions de Barthes, il ne discute que peu philosophiquement avec lui, sans doute parce que la force active, de nature philosophique, de l'œuvre de Barthes est trop importante pour un article. On peut cependant, à la lecture de Labia, commencer de lister ce qui relève d'une dimension philosophique de l'œuvre entrant en résonnance avec des questionnements possibles de la philosophie de la musique. Citons pêle-mêle des pistes qui font de Barthes un interlocuteur: l'importance et le rôle du corps dans l'interprétation comme dans l'écoute; le rôle tenu par le romantisme allemand dans et pour la pensée musicale; le rapport à la musique de celui qui en parlerait philosophiquement, reposant sur une expérience et donc une pensée de l'expérience ; la réflexion portant sur l'acte de parler (philosophiquement) de la, ou de telle, musique; la question cruciale du langage avec lequel on parle de musique. Labia évoque enfin une possible direction à donner à l'abord de la musique par la philosophie en suggérant, selon une proposition rencontrée chez Barthes lui-même ${ }^{25}$, de " renoncer un instant tout aussi bien à la musique "en soi" qu'à celle "pour tous" » (p. 351), c'est-à-dire, de nouveau, de changer «l'objet musical ».

Pour continuer d'explorer comment pense Barthes et comment penser avec lui la musique, on peut revenir au texte de Corbier, parce qu'il pose les jalons d'une histoire de l'objet musique chez Barthes, à la fois linéaire et entrecroisée (d'un mémoire datant de 1941 à 1978), et montre que cette évolution va de pair avec celle du «statut du théâtre et de l'image dans sa réflexion » (p. 81); Corbier suit notamment un objet tel que la choreia (la part de musique qui y entre) dans la pensée de Barthes, le rôle dont elle est investie, puis les changements de perception qu'elle subit, en particulier au contact du Japon, étape cruciale dans la progressive mise en avant de la voix par Barthes (voir les p. 80-88). Corbier produit de surcroît une réflexion stéréophonique grâce à laquelle on constate que Barthes pense avec la modernité, et y conjoint d'autres manières; que l'entremêlement d'une interrogation sur le Texte et d'une modalité renouvelée d'évaluation de la musique s'inscrivent sans rupture « dans le cheminement du sémiologue »; ou encore, que varie la prégnance du regard ou de l'écoute dans la hiérarchie sensitive de Barthes, liée à la force de conducteur intellectuel allouée à la vue ou à l'ouïe selon les périodes.

\section{Écriture}

Mathieu Messager propose une rencontre qui n'eut jamais lieu, celle de Barthes et Pascal Quignard, et produit une approche comparée dessinant «les linéaments d'une conceptualisation assez proche de la musique»(p.327), principalement en ce qui concerne la "valeur singulière de la "voix" ", comme "symbole d'une perte, plurielle et étagée » (p.328). Perte dont Messager montre qu'elle n'est pas seulement de nature déceptive. Pour Barthes, ce que Messager lit comme une "jouissance de la perte", "se dit positivement par l'intermédiaire de la théorie textualiste ; c'est en elle qu'il trouve le ferment pour penser la musique en termes d'érotique ou d'immédiateté qui reste hors métalangage » (p. 331). Or, certes, le piano n'est pas pour Barthes un objet théorique, et il y a beaucoup dans la musique qui échappe à la prise du métalangage, et pourtant, Barthes parle de la 
musique en accommodant un vocabulaire et des idées issus de la théorie du Texte, et parle de ce dernier avec la musique, active des éléments de théorie musicale et d'histoire de la musique dans la pensée littéraire (le texte de Corbier en donne une illustration détaillée, avec le contrepoint et l'articulation entre thème et variations, voir les p. 89-94); comme si écrire sur réclamait de passer par autre chose. Dans ces échanges et jeux d'aller-retour, on rencontre la recherche d'un équilibre, jamais définitif, jamais fixé (ce pourquoi dialogues et oscillations entre métalangage et mise en doute de la capacité du langage à dire l'aimé évoluent mais perdurent), entre irréductibilité du goût, part d'impossible et tentative inlassable de dire. L'écriture de la musique, parler de, parler sur, savoir s'il est possible, et comment, de parler de la musique et plus largement d'une œuvre, constituent un problème qui peut donc intéresser conjointement musicologues et barthésiens. C'est là un dénominateur commun à nombre de contributions de Barthes et la musique, parce que c'est l'une des grandes questions de Barthes, qui constate: «il est très difficile de conjoindre le langage, qui est de l'ordre du général, et la musique, qui est de l'ordre de la différence $^{26} »$; la solution n'est pas dans l'abandon au silence ou à l'ineffable, mais dans la tentative : « le système du langage et la valeur asystématique de la singularité doivent [...] se confronter » constate Patrick Ffrench comme préliminaire à son propos (p. 246 ; je souligne). D'où se révèle l'un des intérêts de la lecture de Barthes parlant de musique, de tentative de et d'invite à travailler le discours que l'on va porter sur la musique. Nommick, par exemple, montre comment "écrire sur la musique » est un problème commun à Barthes et Jankélévitch : pour ce dernier, l'« ineffable déclenche en l'homme l'état de verve » (cité p. 297), et l'on a vu que Barthes propose de changer l'objet - c'està-dire le point de vue depuis lequel on l'aborde -, de « déplacer la frange de contact de la musique et du langage ${ }^{27}$ "; à ces questions, tous deux développent des solutions pratiques voisines.

Sur ce point crucial, la contribution de Christophe Corbier fournit encore d'importants éléments, en proposant indirectement une véritable réflexion sur la critique musicale, qui vaut aussi pour, du moins renseigne sur, la critique - littéraire, picturale et théâtrale, tous types pratiqués par Barthes.

peut-on « écrire la musique » sans écrire « sur » la musique, sans accomplir la tâche du critique qui utilise un métalangage pour parler «sur » un texte? Comment ne pas trahir par le langage une expérience dont les modalités ressemblent à la lecture $\mathrm{du}$ " texte de jouissance » et à l'expérience de «l'inexprimable amour » [Fragments d'un discours amoureux, OC, V, p. 129]?

La proposition "sur » est de toute évidence la clé du problème en ce que sa présence introduit une distance, et même un "distancement", tandis que sa suppression permettrait l'atténuation du clivage langage/musique, analogue au clivage langage/amour. (p. 78)

C'est dans cette contribution, où il est le plus question de la subjectivité, que la démonstration de la validité intellectuelle des idées barthésiennes qui lui sont attachées (adjectif, jouissance) est la plus rigoureuse. Ce qui n'est pas un hasard, puisque Corbier travaille à montrer les valeurs en jeu dans le discours sur la musique, et que, pour Barthes, «commenter la musique, c'est affirmer la valeur sur laquelle repose l'évaluation proposée par le critique» (p. 79): si les valeurs sont subjectives, leur exposé, préalable à tout commentaire, est condition éthique de la validité de celuici. 


\section{Avec la musique}

Enfin, il est une autre grande pratique barthésienne qu'abordent, directement ou indirectement, les contributeurs de Barthes et la musique : penser avec la musique. Pour travailler la barthésienne « tension entre deux forces opposées : l'évaluation singulière et l'analyse métalinguistique ", Patrick Ffrench convoque le "concept " d'acousmatique (le mot désigne un bruit dont on ne voit pas la cause ni/ou l'origine, et renvoie désormais bien sûr aux propositions de Pierre Schaeffer). "Pas décisif dans l'isolement formel de l'objet » (p. 246), l'acousmatique est mise en travail au contact de ce qu'est la voix pour Barthes. L'intérêt de l'essai réside moins, peut-être, dans les «variantes de la situation acousmatique » que Ffrench trouve dans l'œuvre de Barthes - il montre d'ailleurs que la voix-selon-Barthes ne se plie pas au "scénario acousmatique »-, que dans l'ouverture d'une perspective: celle d'approcher, de décrire, quelque chose d'une méthode intellectuelle de Barthes. Il me semble en effet que l'acousmatique permet d'entrevoir une manière de penser, qui dépasse la seule analyse musicale, mais que l'exercice et la pensée de la musique orientent. Avec l'acousmatique, il est question - Ffrench l'a bien vu, qui parle du "geste épistémologique" de Schaeffer - d'attitude dans l'appréhension de l'objet: pour Schaeffer, l'acousmatique, et plus particulièrement l'enregistrement et la "pure écoute » qu'il permet d'un "signal physique » répété, aboutissent à poser «l'objet sonore comme une perception digne d'être observée pour elle-même ${ }^{28}$ ». «L'occultation des causes [...] devient un préalable, une mise en condition délibérée du sujet ${ }^{29}$ ", « l'acousmatique correspond à un renversement du parcours »- l'on pourrait notamment croiser ces modalités avec celles impliquées par le déplacement préconisé dans "Le grain de la voix » cité plus tôt. La communauté formée par Schaeffer et Barthes réside donc dans la démarche qu'implique le changement de point de vue : « la situation acousmatique [...] nous rapproche de l'acte d'écoute et fonctionne donc comme un déplacement paradigmatique dans l'analyse de l'objet musical» (p. 247); avec l'acousmatique, il est possible de travailler concrètement une position critique visà-vis de l'objet.

41 «Peut-être qu'une chose ne vaut que par sa force métaphorique; peut-être que c'est cela la valeur de la musique : d'être une bonne métaphore » écrit Barthes en $1978^{30}$, et beaucoup de contributeurs relaient ce qui doit être lu comme une proposition fertile, mais non une vérité unique, car cette option limiterait beaucoup la description des rapports entre Barthes et la musique. Écœur remarque que «le transit de la figure [de l'amateur] nous indique que la musique elle-même, irréductible à un comparant à vocation purement explicative, prend une fonction initiatrice dans des réflexions qui la dépassent de beaucoup » (p.183); Corbier signale que la musique se trouve souvent chez Barthes "sur une ligne de crête entre métaphore et exemple» (p. 91); dans l'analyse de Baldwin, la métaphore n'est pas que métaphore, notamment parce qu'elle sert aussi à parler de la destruction de la métaphore, et donc se travaille elle-même, selon une énergie qui n'est pas seulement métaphorique; quand Balanche distingue chez Barthes le discours sur la musique du discours sur la lecture, c'est à raison je crois, parce que cela permet de montrer de manière marquante la proximité, les nuances et la possibilité de comprendre, d'éclairer, de renouveler les pensées sur lecture et musique par leur rapprochement sans identification - par un rapport qui fait de la musique plus et autre chose qu'une métaphore. 

nomme une "ressource figurale ", et remarque que Barthes " approche au plus près la rationalité affective et psychique de la vie avec et par la musique » (p. 53). Si la musique est bien un/du matériel pour penser (notamment peut-être selon un processus métonymique de transposition à d'autres objets de pensée), il faut retenir la diversité des rôles qu'elle tient dans la production de Barthes, et surtout, l'imbrication de ceuxci. La musique est donc, me semble-t-il, toujours plus qu'une métaphore, à la fois parce que son rôle est autrement quotidien et fondateur, et parce qu'elle est pour Barthes une réserve poḯtique qui ne relève pas que de l'image ou de l'imagé, ce que certaines contributions du volume Barthes et la musique montrent sans le souligner. produire intellectuellement pour la musique ce que Barthes défend en parlant du corps, à savoir une "extension un peu métonymique » de l'objet, permettant d'en saisir ensemble tous les enjeux - et de comprendre qu'il y a chez Barthes non seulement des problématiques propres à la musique et d'autres à la littérature, mais encore qu'il est des problématiques barthésiennes communes à la musique et à la littérature, nées de la manière singulière dont il aborde l'une et l'autre. Peut-être parce qu'il a été lu plutôt du côté de Barthes que de celui de la musique, Barthes et la musique me semble principalement renseigner sur ce que peut faire la musique aux études barthésiennes; et pourtant, comment parler de musique, dire la littérature, «comment faire se succéder les thèmes dans le temps " (Corbier p.91), comment procéder à une interprétation, comment évaluer une interprétation, sont autant de questions pratiques qui entretiennent musicologues et musiciens de leur propre pratique.

Enfin, si le goût barthésien implique une solitude (ou plutôt deux : être le seul à aimer, être le seul à jouer), notons que la pratique solitaire de l'amateur ouvre sur une communauté intéressante à penser: "ce "moi", "vrai pianiste schumannien", c'est Barthes lui-même, schumannien "pratiquant", mais c'est aussi celui qui joue Schumann, quel qu'il soit, au moment où il le joue » (Fabre, p. 62), et, de cette manière, la sienne, propre à chacun, comprend l'œuvre: si l'expérience est solitaire, elle est cependant commune. Il apparaît que faire de la musique autrement et proposer une production autrement savante vont de pair chez Barthes. Ne pas éteindre ses goûts propres, traiter au contraire de ses propres goûts, forment un impératif barthésien, dans la mesure où par eux seuls il est possible de travailler à leur transmission, même partielle.

\section{NOTES}

1. BARTHES Roland, «Le grain de la voix» (Musique en jeu, novembre 1972), OC, IV, p. 149. Les références aux textes de Barthes renverront désormais aux Euvres complètes en cinq tomes, éd. Éric Marty, Paris, Seuil, 2002 (OC, suivi de la tomaison et de la pagination) ; les références sans autre indication renvoient à Barthes et la musique. 
2. Les principaux textes sur la musique de Barthes ont été réunis de manière posthume dans la seconde section de L'obvie et l'obtus. Essais critiques III ; cependant, aux lecteurs intéressés on conseille de se référer aux Euvres complètes, qui permettent de suivre les évolutions et glissements intellectuels de Barthes, et de lire, en regard d'un texte touchant à la musique, la production de la période à laquelle celui-ci a été écrit.

3. Sur ce point, voir coste Claude, «Barthes: musique au présent», Orphée ou les sirènes. L'imaginaire littéraire de la musique, Paris, Presses universitaires de Paris Ouest, 2014, p. 76-77.

4. ESCAL Françoise, "Roland Barthes: Fragments d'un discours sur la musique ", initialement paru dans Semiotica, vol. 66, $\mathrm{n}^{\circ} 1 / 3,1987$, p. 57-68 et republié, avec une présentation d'Esteban Buch, dans le Hors-série $\mathrm{n}^{\circ} 1$ de Transposition. Musique et sciences sociales "Musique, histoire, sociétés. Les études sur la musique à l'EHESS ", 2018 (https://journals.openedition.org/transposition/1715, consulté le 27 mai 2019).

5. Il y a chez Barthes une représentation de la dynamique du goût, mêlant la conscience de sa nature culturelle à une libre et syncrétique appropriation de Michelet et Nietzsche, le tout façonnant une représentation faisant dépendre le goût d'un système idiosyncrasique d'attractions et de répulsions.

6. Celle-ci est citée dans cosTE et DOUCHE, Barthes et la musique, p. 21.

7. BARTHES, « Le grain de la voix », p. 149.

8. Le géno-chant est pour Barthes «le volume de la voix chantante et disante [...]; un jeu signifiant étranger à la communication [... ;] cette pointe (ou ce fond) de la production où la mélodie travaille vraiment la langue - non ce qu'elle dit, mais la volupté de ses sons-signifiants, de ses lettres ". Le phéno-chant, lui, « couvre tous les phénomènes, tous les traits qui relèvent de la structure de la langue chantée, des lois du genre, de la forme codée du mélisme, de l'idiolecte du compositeur, du style de l'interprétation ». BARTHES, « Le grain de la voix », p. 150-151.

9. Ibid., p. 155.

10. BARTHES Roland, «Le chant romantique » (texte dit sur France Culture en mars 1976, publié dans Gramma en janvier 1977), OC, V, p. 305.

11. Voir BARTHES Roland, «Musica practica " ( $L^{\prime} A r c n^{\circ} 40$, février 1970, pour un numéro sur Beethoven dirigé par Boucourechliev), OC, III, p. 447-450.

12. «Dans son état utopique, la langue serait élargie, je dirais même dénaturée, jusqu'à former un immense tissu sonore dans lequel l'appareil sémantique se trouverait irréalisé ; le signifiant phonique, métrique, vocal, se déploierait dans toute sa somptuosité, sans que jamais un signe s'en détache (vienne naturaliser cette pure nappe de jouissance), mais aussi - et c'est là le difficile - sans que le sens soit brutalement congédié, dogmatiquement forclos, bref châtré. [...] la langue ne quitterait pas pour autant un horizon du sens: le sens, indivis, impénétrable, innommable, serait cependant posé au loin comme un mirage, faisant de l'exercice vocal un paysage double, muni d'un "fond"; mais au lieu que la musique des phonèmes soit le "fond" de nos messages (comme il arrive dans notre Poésie), le sens serait ici le point de fuite de la jouissance. » BARTHES Roland, «Le bruissement de la langue » (Vers une esthétique sans entraves. Mélanges Mikel Dufrenne, 1975), OC, IV, p. 801-802.

13. BARTHES, « Le grain de la voix », p. 153.

14. COSTE et DOUCHE, Barthes et la musique, p. 21.

15. BARTHES, «Le grain de la voix », p. 148 puis 149.

16. BARTHES Roland, « Rasch » (Langue, discours, société. Pour Émile Benveniste, Paris, Seuil, 1975), OC, IV, p. 827.

17. Pour une vue d'ensemble, et la démonstration de la force d'une telle figure, qui doit être pensée au croisement des disciplines et pratiques barthésiennes, on peut aussi se reporter à CHASSAIN Adrien, "Roland Barthes: "Les pratiques et les valeurs de l'amateur" ", Fabula-LhT, n 15, octobre 2015 (http://www.fabula.org/lht/15/chassain.html, consulté le 27 mai 2019). 
18. BARTHES Roland, Journal de deuil. 26 octobre 1977-15 septembre 1979, éd. Nathalie Léger, Paris, Seuil/Imec, 2009.

19. BARTHES Roland, Avant-propos à Sur Racine (1963), OC, II, p. 55.

20. BARTHES Roland, « Histoire ou Littérature ?» (Sur Racine), OC, II, p. 193.

21. BARTHES Roland, Critique et vérité, Paris, Seuil, 1966, p. 75

22. Pour tout cela, voir BARTHES Roland, «Écoute » (pour l'Encyclopédie Einaudi, écrit avec Roland Havas, 1977), OC, V, p. 340-352.

23. Dont Barthes fait à l'occasion un " performateur », voir « Musica practica », p. 450.

24. "Si nous réussissions à affiner une certaine "esthétique" de la jouissance musicale, nous accorderions sans doute moins d'importance à la formidable rupture tonale accomplie par la modernité ». BARTHES, « Le grain de la voix », p. 156.

25. Voir BARTHES Roland, «La musique, la voix, la langue » (conférence à Rome, mai 1977), OC, V, p. 523 et $s q$.

26. Ibid., p. 524.

27. BARTHES, « Le grain de la voix », p. 149.

28. SCHAEFFER Pierre, Traité des objets musicaux. Essai interdisciplines, Paris, Seuil, 1966, p. 94.

29. Ibid., p. 92.

30. Dans BARTHES, « La musique, la voix, la langue », p. 528.

\section{AUTEURS}

\section{CÉCILE RAULET}

Cécile Raulet prépare, sous la direction de Philippe Roger à l'EHESS (CRAL), une thèse sur "L'ethos du critique littéraire », et plus particulièrement sur la présence et l'éthique critiques de Roland Barthes. Elle cherche à rendre compte des gestes de la critique et à traiter de la présence du critique dans et à son texte ; cela passe par une attention pour les formes du dire, en tant que l'écriture est à la fois force de transmission de l'expérience d'une œuvre et condition de cette transmission. Il s'agit donc de décrire une éthique de Barthes, véhiculée par l'écriture même, qui diffuse des principes en étant fidèle à ceux-ci dans le moment où elle puise dans les ressources du langage. Enfin, parce que des tournures de phrases disent aussi des tournures d'esprit, Cécile Raulet espère dégager certaines conformations de pensée, modalités éthiques de réflexion à partir de cette lecture d'un esprit critique à l'acte, s'exerçant sur, par et dans l'écriture. Hormis une activité de relectrice-correctrice de textes littéraires et universitaires, la participation au comité de rédaction de la Revue Roland Barthes en ligne et au Dictionnaire Barthes (à paraître), on peut signaler la contribution « Considérer ce qui revient » dans Roland Barthes : continuités (Christian Bourgois, 2017), ainsi que sa traduction en italien pour la revue Logos. Rivista di filosofia, $\mathrm{n}^{\circ} 13,2018$. 